0,85 Grm. gaben 0,3235 $(\mathrm{MgO})^{2} \mathrm{PO}_{3}=0,0923 \mathrm{P}$.

$0,6205 » \quad 1,483 \quad \mathrm{Ag} \mathrm{Cl}=0,3665 \mathrm{Cl}$.

$0,5 \gg \quad 0,579 \quad \mathrm{BaO} \mathrm{S0} \mathrm{S}_{3}=0,0794 \mathrm{~S}$.

Die procentische Zusammensetzung dieser Verbindung ist demnach :

$\mathrm{P}=10,86$ die Formel : $\mathrm{P} \mathrm{Cl}_{5} 3 \mathrm{SO}_{2}$ giebt 10,48

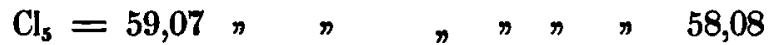

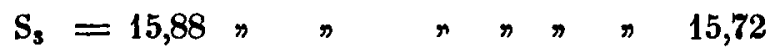

$0_{6}=\frac{14,19}{100,00} \gg \geqslant \geqslant \geqslant \frac{15,72}{100,00 \text {. }}$

Sie unterscheidet sich nur durch geringeres spec. Gew. und Lichtbrechungsvermögen von der vorigen.

Ammoniak, über schwefligsaures Phosphorchlorid geleitet, giebt dieselben Erscheinungen, die man erhält, wenn man seine Bestandlheile für sich mit Ammoniak behandelt. Man erhält nämlich Salmiak, trocken zweifach sclıwefligsaures Ammoniak und ein weifses Pulver, das sich von dem durch Behandlung von Phosphorchlorid mit Ammoniak erhaltenen nicht unterscheidet. Eine vorläufige Prüfung der bei $100^{\circ}$ getrocknelen Substanz gab indefs nur 36,18 pC. Phosphor.

\title{
Ueber die einfachen Platincyanverbindungen;
}

\section{von Bernard Quadrat.}

Im 63. Bande Seite 164-194 dieser Annalen habe ich die Resultate meiner Untersuchung ïber Platincyanverbindungen veroffentlicht. Ich begann die Untersuchung der Platincyanverbindungen in der Absicht, die dem Gmelin'schen Kalisalze entsprechend zusammengesetzten Verbindungen darzustellen und ihre Eigenschaften näher zu studiren. Die unlängbar miihsame und im Grunde genommen auch nicht schr ausgiebige Darstellungsart 
miltelst Blutlaugensalz und Plalinschwamm nölhigte mich, von der von mir (in diesen Annalen Band 63 Seite 167) angegebenen Methode Gebrauch zu machen. Durch den dabei angewandten Ueberschufs von Cyankalium erzielt man aber die Bildung des nach der Formel : $\mathrm{Pt}_{\mathbf{3}} \mathrm{K}_{6} \mathrm{Cy}_{11}$ zusammengesetzten Kalisalzes. Dieses Salz krystallisirt, wie oben bemerkt (Bd. 63 S. 167) ausnehmend leicht und nach 2-3maligem Umkrystallisiren erhält man dasselbe rein und nach oben angeführter Formel zusammengesetzt. Dieses Kalisalz $\left(\mathrm{PI}_{5} \mathrm{~K}_{6} \mathrm{Cy}_{11}\right)$ ist nicht ein Gemenge von dem einfachen Kalisalz ( $\mathrm{Pt} K \mathrm{KC}_{2}$ ) und $\mathrm{K} \mathrm{Cy}$.

Stellt man aus demselben die übrigen Verbindungen dar, so erhält man Salze von der Zusammenselzung $\mathrm{Pt}_{5} \mathrm{M}_{0} \mathrm{Cy}_{11}$ (wo $M$ das entsprechende Metall vertritt).

Kocht man das $\mathrm{Pt}_{5} \mathrm{~K}_{6} \mathrm{CY}_{11}$ lange Zeit hindurch mit Wasser, so erlualt man nach öfterem Umkrystallisiren Verbindungen, deren Platingehalt je nach der öfteren Unkrystallisation stets höher steigl, bis derselbe endlich das Maximum 51,98 pC. erreicht.

Ich erhielt Kalisalze, deren Platingehalt, wie folgt, durch Unkrystallisiren immer zunahm.

Die erste Umkrystallisation gab 49,05 pC. Pt

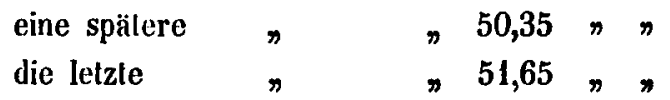

Die Formel : Pt $\mathrm{K} \mathrm{Cy}_{2}$ erheischt 51,98 pC. Platin.

In dem Bd. 65 S. 250 ist angeführl, dafs die aus dem grünen Platincyankupfer miltelst Schwefelwasserstoff dargestellte Säure schwefelhaltig sey und nach Sälligung mit einer Basis Eisenoxydsalze blutroth färbe und dafs die damit dargestellten Salze meistens eine Schwefelcyanverbindung enthalten. Die genaueste Untersuchung hat mir jelzt dargethan, dafs die von mir in meiner crsten Abhandlung beschriebenen Salze frei sind von einer Einmischung einer Schwefelverbindung; und ich besilze selbst eine Platincyanwasserstoffsäure, welche, aus einem zusammengeseizten Kupfersalz dargestellt, keine Spur von einer 
Schwefelcyanverbindung enthält. Das soeben bescliriebene Verhalten des Kalisalzes der Reihe : $P_{5} C_{Y_{11}} K_{6}$ zeigt deullich, dafs es eine Doppelverbindung einer höheren Ordnung ist.

lch bin der Ansicht, dafs nicht zwei (wie ich durch analyt. Resultate bereils zum Theil früher bewiesen habe), ja dafs noch mehrere Reihen von Platincyanverbindungen existiren.

Den Gegenstand vorliegender Abhandlung bilden einige Salze der einfachen Cyanplatinreihe, und zwar das Kali-, Natron-, Kalk-, Baryt-, Magnesia- und Kupfersalz, die ich in Hrn. Professor Redtenbacher's Lajoratorium untersuchte.

\section{Kalisalz.}

Die Darstellung ist im Vorhergehenden bereits beschrieben, ich führe hier blol's die erhaltenen analytischen Resultate an:

1,419 Grm. bei $280^{\circ}$ getrockneten Salzes gaben nach vorhergrgangenem Behandeln mit Schwefelsäure 0,733 Grm. Platin $=51,65 \mathrm{pC}$. Platin, woraus sich das gefundene Atomgewicht mit 2386 berechnet. Das berechnete Atom ist 2372 und verlangt 51,98 pC. Platin.

Ferner gaben 0,569 Grm. derselben Substanz 0,261 Grm. schwefelsaures Kali, welches $20,60 \mathrm{pC}$. Kalium entspricht.

Das Cyan berechnet sich aus dem Verlusle mil 27,75 pC.

\begin{tabular}{|c|c|c|c|c|}
\hline \multicolumn{3}{|c|}{ Versuch } & \multicolumn{2}{|c|}{ Rechnung } \\
\hline $\mathbf{P t}$ & 51,65 & $\mathrm{Pt}$ & 1233,0 & 51,98 \\
\hline & 20,60 & $\mathbf{K}$ & 488,9 & 20,62 \\
\hline$y$, & 27,75 & $\mathrm{Cy}_{\mathrm{y}_{2}}$ & 650,0 & 27,40 \\
\hline & $\begin{array}{l}\text { berechnetes } \\
\text { gefundenes }\end{array}$ & $\begin{array}{c}\text { Atomgewicht } \\
n\end{array}$ & $\begin{array}{l}2371,9 \\
2395 .\end{array}$ & 100,00 \\
\hline
\end{tabular}

\section{Natronsalz.}

Durch Kochen des Platincyankupfers in Ueberschusse mit kohlensaurein Natron, Fltriren und Abdampfen, erhält man grofse Krystalle des Natronsalzes. Die farblosen durchsichtigen Krystalle, 
dem hemiprismatischen Systeme angehörend, erinnern an die bekannten Augilformen, sie zeigen die Grundgestalt combinirt mil dem vertikalen Prisma und dem mikrodiagonalen Flächenpaar, wozu oft auch das mikrodiagonale horizontale Prisma tritt. Durch starke Entwicklung des mikrodiagonalen Flächenpaars sind meistens die Prismen tafelartig. Hemitropische Zwillingskrystalle finden sich mitunter. Die Spaltbarkeit parallel zur Grundfläche ist ausgezeichnet. Die Spaltungsflächen zeigen starken Glasglanz.

Die Krysialle sind in Wasser, sowie auch in Alkohol löslich. Mit einer Auflösung von salpetersaurem Quecksilberoxydul giebt das Cyanplatinnatrium sebr oft einen hochrothen Niederschlag.

Bei der Analyse gaben 0,850 Grm. bei $280^{\circ}$ getrockneter Substanz 0,480 Grm. Platin $=56,53$ pC. und 0,454 Grm. schwefelsaures Natron $=13,10 \mathrm{pC}$. Natrium.

Das Cyan ergiebt sich aus dem Verluste mit $30,37 \mathrm{pC}$.

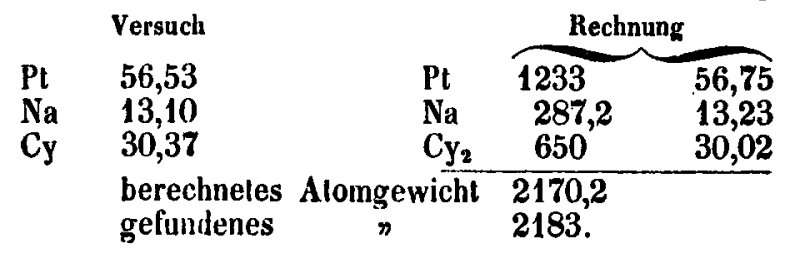

\section{Kalksalz.}

Die Darslellung des Platincyancalciums beruht auf der Zersetzbarkeit des Kupfersalzes durch Aetzkalk bei der Kochhitze des Wassers. Die vom ausgeschiedenen Kupferoxyd abfiltrirte Flüssigkeil wird durch Einleiten von Kohlensäure und nachherigem Erhilzen von dem überschüssigen Aetzkalk befreit. Verdampft man die Flüssigkeit, so krystallisirt das Kalksalz beim Erkalten in dünnen hemiprismatischen Nadeln. Die Krystalle zeigen denselben Trichroismus wie das Barylsalz, cilronengelb und zeisiggrün im durchfallenden, bläulich diantantglianzend im auffallenden Lichte. 
Die Krystalle sind in Wasser löslich; bei einer Temperatur von $100^{\circ} \mathrm{C}$. werden sie anfangs rolhbraun, dann blau. Bei $180^{\prime \prime}$ werden sie gelb.

$0,932 \mathrm{Grm}$. lufturocknen Kalksalzes verl. bei $180^{\circ} 0,190 \mathrm{Grm}$. Wasser. Dieses entspricht 20,38 pC. Krystallwasser.

$0,742 \mathrm{Grm}$. bei $180^{\circ}$ getrockneter Substanz gaben 0,427 Grm. Platin $=57,55$ pC. und 0,215 Grm. kohlensauren Kalk, entsprechend $11,56 \mathrm{pC}$. Calcium.

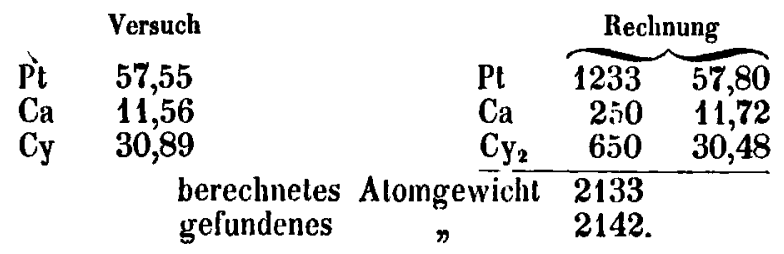

Versetzt man die Auflösung des Platincyancalciums mit einer Iösung von Chlorcalcium im Ucberschusse, so erhält man beim Abdampfen klare, glänzende, sechsseilige Prismen des prismatischen Systems, von blafs grünlichgelber, längs der Axe intensiv zeisiggrüner Durchsichligkeitsfarbe, aus den Prismaflächen lichtblauer Diamantglanz. Es sind dicse Kryslalle eine Verbindung von Platincyancalcium mit Chlorcalcium.

\section{Barytsalz.}

Durch Kochen mil Aelzbaryt wird das Kupfersalz derart zerlegt, dafs an die Stelle des Kupfers Barium tritt und wasserfreies Kupferoxyd sich abscheidet. Durch Filtriren und Einleiten von Kohlensäure entfernt man das Kupferoxyd, sowic auch den überschuissig zugesetzlen Baryt. Bein Abdampfen der Flüssigkeil schiefsen Krystalle des Barytsalzes an.

Sechsseilige Prismen mit Endfläche, hemiprismatisch :

$$
P+\infty \cdot P-\infty \cdot \overline{\operatorname{Pr}}+\infty \text {. }
$$

'Tiefcilrongelb, durchsichtig, auf den Prismaflächen violeltblaues Schillern. In der Axenrichlung zeigen dic lirystalle lichles Gelbgrün als Durchsichligkeilsfarbe. 
Die Krystalle sind in heifsem Wasser löslicher als in kaltem, bei $140^{\circ}$ werden dieselben orange, mit einem Stich in's Braune, dann grünlich und zuletzt weils.

Das Krystallwasser beträgt $15,3 \mathrm{pC}$.

0,742 Grm. bei $180^{\circ}$ getrockneten Salzes gaben, in Wasser gelöst und mit Schwefelsäure verselzt, 0,394 Grm. schwefelsauren Baryt $=31,25$ pC. Barium.

Das Platin wurde aus einer andern Quantitit Salzes beslimmt, und zwar wurden auf 1,170 Grm. Substanz 0,523 Grm. Platin $=44,70 \mathrm{pC}$. entsprechend erhallen.

Der procentische Verlust ergiebt die Menge des in ter Verbindung enthallenen Cyans $=24,05 \mathrm{pC}$.

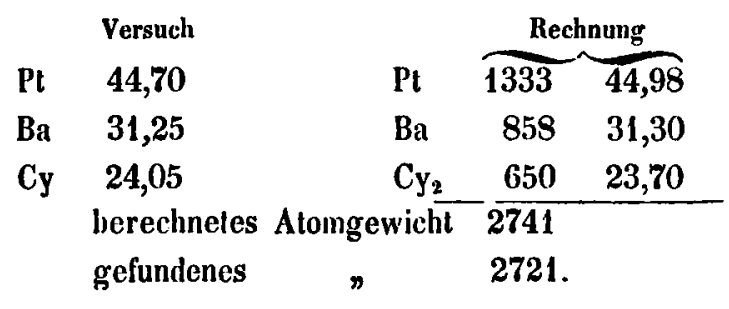

Magnesiasals.

Das nach der Formel : Pt $\mathrm{Mg} \mathrm{Cy}_{2}$ zusammengeselzle Salz wurde nach der von mir für das $\mathrm{Pt}_{3} \mathrm{Mg}_{6} \mathrm{Cy}_{11}$ angegebenen Methode (diese Annalen Bd. 63 S. 175) dargestell; jedoch nahm ich statt Aelheralkohol, rectificirten Weingeist. Ich hatle sehr oft Gelegenheit, die Bildung verschieden gefärbler Krystalle zu bemerken. War die Lösung in Alkohol concentrirt, so erschienen im Beginn des Krystallisirens ungefärbte durchsichlige Nadeln, welche in demselben Maafse als der Alkohol verdunsteten, schwefelgelb wurden und sich endlich in fleischrolhe Irrystalle verwandelten.

Bei der Krystallisation findel eine, jedoch unbedeutende, Abscheidung eines bräunlichen Körpers slatt. 
Läfst man eine heils gesälligle wässerige Lösung des Salzes erkalten, so bilden sich blutrolhe Krystalle.

Die Iírystallform ist dieselbe, welche das $\mathrm{Pt}_{5} \mathrm{Mg}_{6} \mathrm{Cy}_{11}$ besilzl. Erhilzt wird es schwefelgeib, später braun.

$0,576 \mathrm{Grm}$. bei $280^{\circ}$ getrockneter Substanz gaben durch Glïhen mit Schwefelsüure 0,346 Grm. Platin $=60,07$ pC. und $0,2133 \mathrm{Grm}$. schwefelsaurer Magnesia $=7,71 \mathrm{pC}$. Magnesium.

Das Cyan belrägt 32,22 pC.

\begin{tabular}{|c|c|c|c|c|}
\hline \multicolumn{3}{|c|}{ Versuch } & \multicolumn{2}{|c|}{ Hechnung } \\
\hline $\mathrm{Pl}$ & 60,07 & $\mathrm{Pt}$ & 1233 & 60,44 \\
\hline $\mathrm{Mg}$ & 7,71 & $\mathbf{M g}$ & 1577 & 7,73 \\
\hline Cy & 32,21 & $\mathrm{Cy}_{2}$ & 650 & 31,83 \\
\hline & $\begin{array}{l}\text { berechnetes } \\
\text { gefundenes }\end{array}$ & $\begin{array}{c}\text { Alomgewicht } \\
\eta\end{array}$ & $\begin{array}{l}2040,7 \\
2053 .\end{array}$ & \\
\hline
\end{tabular}

\section{Ammoniaksalz.}

Cyanplalinwassersloff isl, wie ich in der ersten Abhandlung über Platincyanverbindung bemerkle, das empfindlichste Reagens auf Ammoniak, wodurch sich derselbe gelb färbt.

Leilet man über bei $100^{\circ}$ getrockneten Plalincyanwasserstoff trocknes Ammoniakgas, jedoch mit der Vorsicht, dafs der Platincyanwasserstoff im Ueberschusse vorhanden ist, so färbt sich derselbe gelb, ein Ueberschurs von Ammoniak zerstört die gelbe Farbe, an deren Stelle die weilse Farbe trilt. An der Luft färbt sich die weifse Verbindung gelb durch Ammoniakverlust und reagirt zugleich sauer.

Versucht man aus Platincyankalium und schwefelsaurem Ammoniak durch Zusammenbringen der entsprechend wässerigen Lösungen, Eindampfen zur Trockne und Ausziehen mit Alkohol das Ammoniaksalz darzustellen, so bilden sich beim Abkühlen der alkoholischen Lösung prismalische Krystalle, welche, so lange sie sich in der Flüssigkeit befinden, farblos, an der almosphärischen Luft sich gelb färben, Ammoniak verlieren und sauer 
reagiren; in eine Ammoniakatmosphäre gebracht, werden dicselben farblos.

\section{Kupfersalz.}

Eine Lösung von Platincyankalium fältt aus IKupfervitriollösung hellgrünes Platincyankupfer, welches alle Eigenschaften mit dem $\mathrm{Pt}_{s} \mathrm{Cu}_{0} \mathrm{Cy}_{11}$, mit Ausnahıne seiner Zusammenselzung, theilt.

Die analytischen Resultale sind, wie folgt :

1,150 Grm. bei $120^{\circ}$ getrockneter Substanz gaben 0,629 Grm. Platin $=54,67$ pC. und 0,249 Grm. Kupferoxyl, entsprechend 17,30 pC. Kupfer.

Die Cyanmenge ist somit $28,03 \mathrm{pC}$.

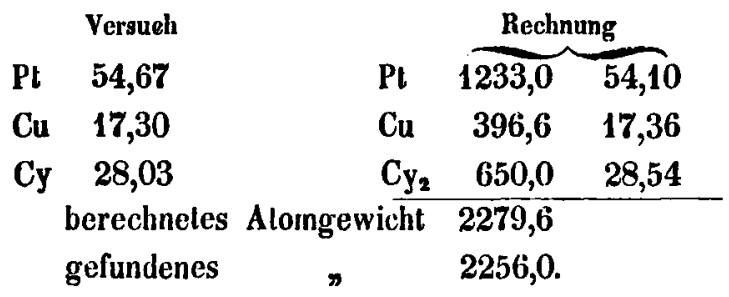

Das Kupfersalz löst sich in Ammoniak auf, aus welcher Lösung durch freiwilliges Verdunsten blaue Krystalle entstehen.

Ist das angewandte Kupfersalz frisch dargesleltt, so erhält man grofse, dicke, lasurblaue Krystalle; war das Kupfersalz trocken, resultiren feine Nadeln.

Es existiren zwei Verbindungen des Platincyankupfers mit Ammoniak; die ammoniakreichere liefert grofse, dicke, lasurblaue; die ammoniakärmere feine, nadelförmige, kornbluınenblaue Krystalle.

Schliefslich bemerke ich, dars ich durch Einleilen von Chlor in die Lösung des Platincyankaliums ( $\mathrm{Pt} \mathrm{K} \mathrm{Cy}_{2}$ ) ein neues Salz, wahrscheinlich das Platincyanidkalium, erhalten habe, mil dessen Untersuchung ich eben beschäftigt bin. 